\title{
One Year On: New and Unique Applications of He Ion Microscopy
}

\author{
D.C. Bell ${ }^{*}$, N. Erdman ${ }^{* *}$, M.A. Jepson ${ }^{* * *}$, C. Rodenburg ${ }^{* * *}$, and B.J. Inkson ${ }^{* * *}$
}

*School of Engineering and Applied Sciences, and Center for Nanoscale Systems, Harvard University, Cambridge, Massachusetts 02138

***JEOL USA Inc, Peabody, MA 01960

** Department of Engineering Materials, The University of Sheffield, Sheffield, U.K.

The Helium ion microscope (HeIM) developed by Zeiss ALIS (Orion microscope) is now commercially one year old and still a unique instrument for imaging and characterization of nanoscale materials and composites [1,2]. The helium ion source has proven to be very stable and offers high brightness, low virtual size and low energy spread as compared to a conventional FEG type SEM, and has low contamination issues. The HeIM microscope offers a remarkable depth of field, compositional analysis ability via RBS system, limited charging effects and excellent surface information on low Z materials.

We will show more recent results applying HeIM imaging in the analysis of semiconducting materials and devices. Some of the results have been surprising and may provide a foundation for a change in analysis techniques of semiconducting materials. The imaging and contrast mechanisms provided by the HeIM are sufficiently different in comparison to standard SEM imaging to provide further details of semiconducting materials that have been previously unobtainable or at the least difficult to acquire. Details of feature imaging, edge detection, sub-surface imaging and dopant imaging will be presented in this paper (Figure 1).

The secondary electron imaging obtained by the HeIM shows enhanced edge contrast due to the nature of the Helium ion beam interactions with the sample. This could be especially useful for critical dimension measurements, and enhance edge detection by an order of magnitude; details of edge measurements of common semiconductor materials and fabricated structures will be shown. Subsurface feature imaging has also been demonstrated using Rutherford backscattered ion imaging (RBI), to allow imaging of the underlying details of fabricated devices. Dopant contrast in the HeIM is also presented and compared to SEM imaging. It is demonstrated that Helium ion microscopy offers improved resolution, but the contrast mechanism is more complex than in the SEM.

The ability to use an electron flood gun in HeIM on samples that traditionally charge up in a SEM also adds to the ability to image previously considered difficult samples (Figure 2). We will show examples of samples traditionally charging in SEM imaged using HeIM, from both biological and materials applications.

\section{References}

[1] D.C. Bell "Contrast Mechanisms and Image Formation in Helium Ion Microscopy", Microscopy and Microanalysis, in press (2009). 
[2]J. Morgan, J. Notte, R. Hill, B. Ward "An Introduction to the Helium Ion Microscope", Microscopy Today (2006), vol. 14 (4), pp. 24-31.

[3] This work was performed in part at the Center for Nanoscale Systems (CNS), a member of the National Nanotechnology Infrastructure Network (NNIN), which is supported by the National Science Foundation under NSF award no. ECS-0335765.
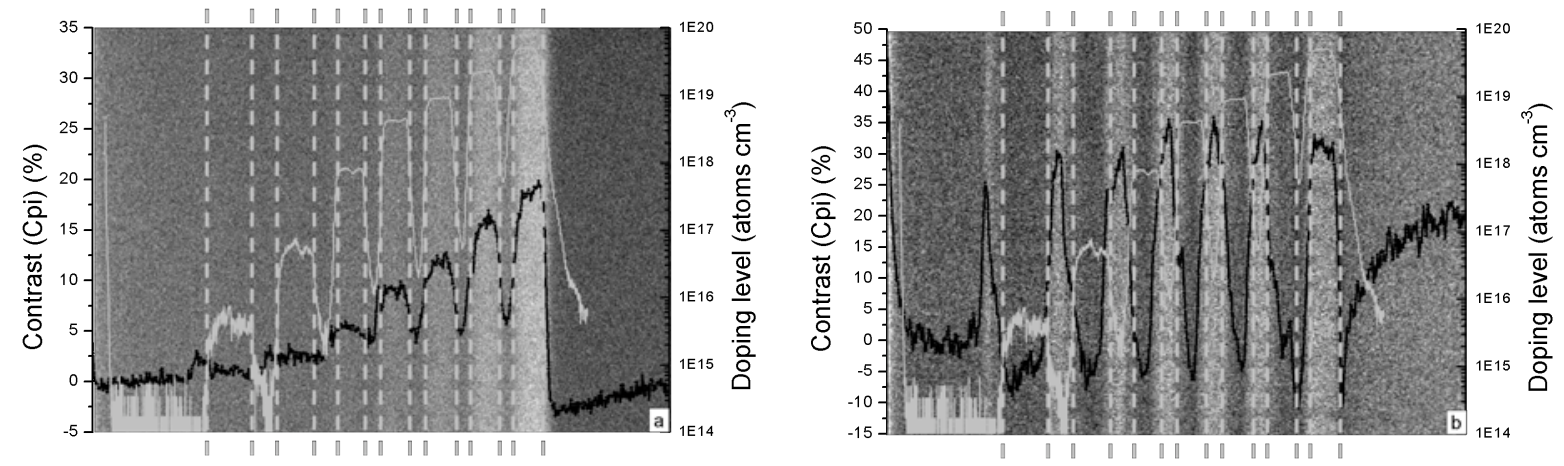

Figure 1. Left - SEM image of a sample at $0^{\circ}$ tilt with contrast (black) and SIMS profile (grey) overlaid. Right - HeIM image with contrast (black) and SIMS profile (grey) overlaid
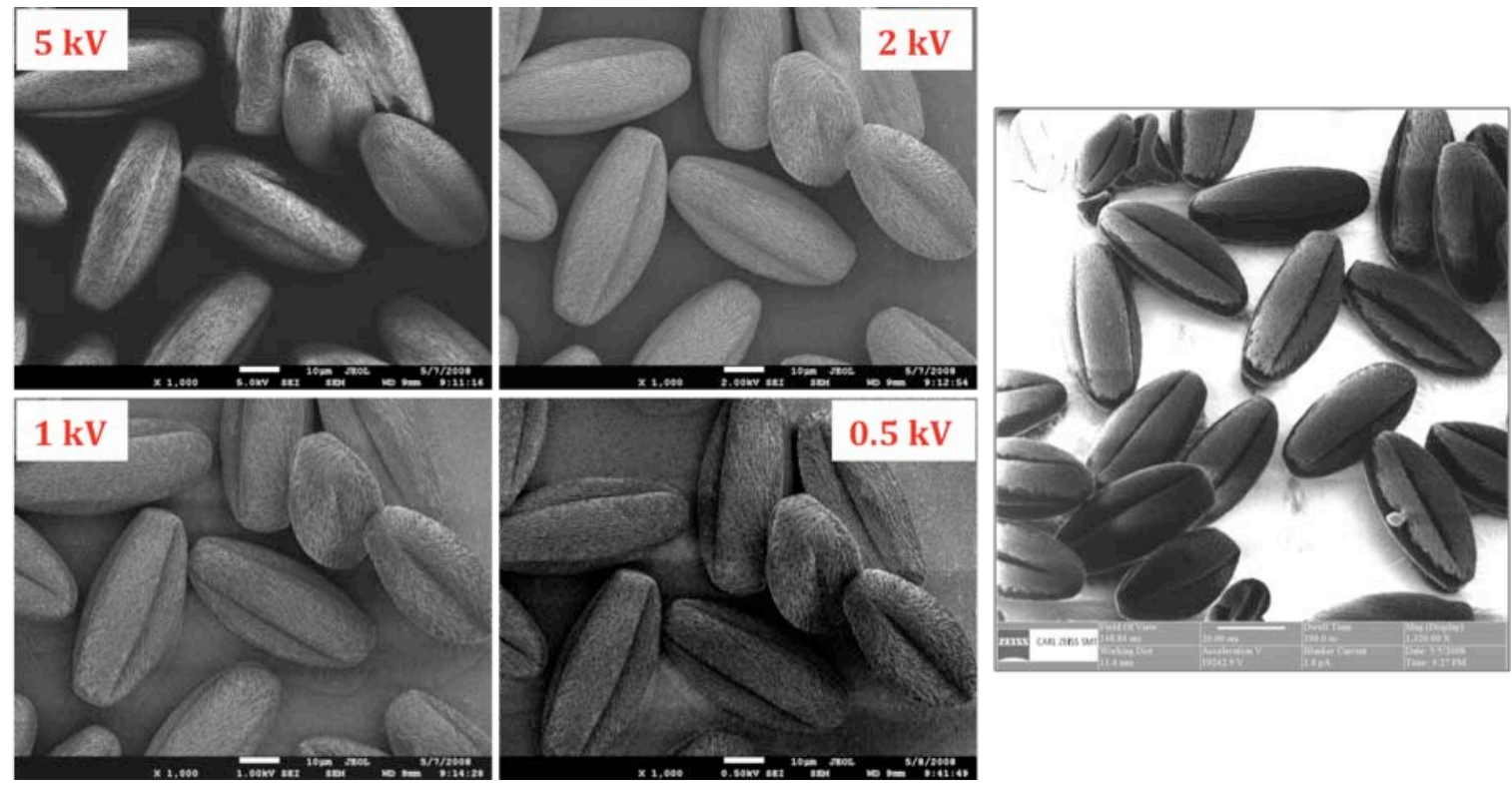

Figure 2. Pollen sample. On the left -4 SEM images, showing accelerating voltage progression to achieve charge balance and contrast reversal upon changing $\mathrm{kV}$; on the right - image of the same sample from the HeIM. 